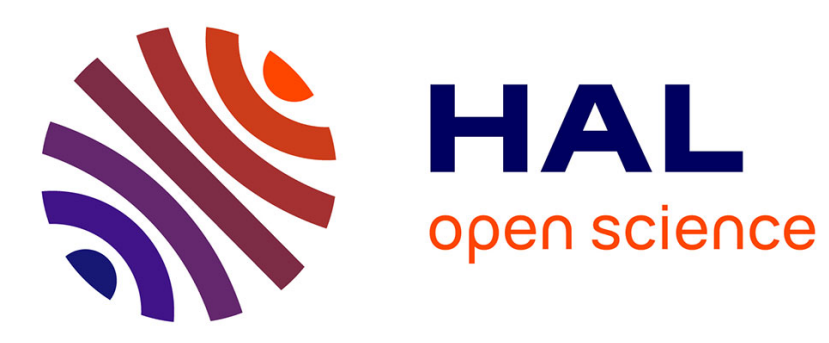

\title{
MIMO Cooperation Schemes for Uplink of Future Railway Communication Systems
}

Karine Amis, Thomas Galezowski, Xavier Lagrange

\section{To cite this version:}

Karine Amis, Thomas Galezowski, Xavier Lagrange. MIMO Cooperation Schemes for Uplink of Future Railway Communication Systems. PIMRC 2020: IEEE 31st Annual International Symposium on Personal, Indoor and Mobile Radio Communications, Aug 2020, London, United Kingdom. 10.1109/PIMRC48278.2020.9217233 . hal-02904429

\section{HAL Id: hal-02904429 \\ https://imt-atlantique.hal.science/hal-02904429}

Submitted on 22 Jul 2020

HAL is a multi-disciplinary open access archive for the deposit and dissemination of scientific research documents, whether they are published or not. The documents may come from teaching and research institutions in France or abroad, or from public or private research centers.
L'archive ouverte pluridisciplinaire HAL, est destinée au dépôt et à la diffusion de documents scientifiques de niveau recherche, publiés ou non, émanant des établissements d'enseignement et de recherche français ou étrangers, des laboratoires publics ou privés. 


\section{MIMO Cooperation Schemes for Uplink of Future Railway Communication Systems}

\author{
Karine Amis \\ IMT Atlantique \\ UMR CNRS 6285 Lab-STICC, \\ Brest, France \\ karine.amis@imt-atlantique.fr
}

\author{
Thomas Galezowski \\ Société du Grand Paris \\ Paris, France \\ thomas.galezowski@societedugrandparis.fr
}

\author{
Xavier Lagrange \\ IMT Atlantique \\ UMR CNRS 6074 IRISA, \\ Rennes, France \\ xavier.lagrange@imt-atlantique.fr
}

\begin{abstract}
In this paper, we consider the uplink of a railway communication system with onboard mobile relays. Each onboard relay communicates with two cooperating base stations at the ends of the section along which the train is moving. Each carriage is equipped with two distinct antenna arrays. We design transmit coding schemes distributed between the antenna arrays and we apply receive macrodiversity. Simulations show that the proposed schemes enable to maintain transmission quality and throughput as the carriage moves along the section.

Index Terms-MIMO systems, railway communications, mobile relay, macrodiversity, distributed coding
\end{abstract}

\section{INTRODUCTION}

Democratization of mobile wireless devices (smartphones, tablets, ...) combined with fast-growing usage of connected machines (Internet of things) makes it necessary for public transport to adapt and to provide high-quality mobile wireless connectivity. Indeed, $4 \mathrm{G}$ quality of service is not satisfactory enough onboard and the throughput may fluctuate.

Among proposed solutions [1], the deployment of onboard mobile relays seems a good compromise to ensure high-quality services to passengers. Société du Grand Paris, in charge of designing and constructing future Grand Paris Express lines (200 km of fully-automated metro lines, 2 million passengers per day) [2], wants to provide continuous high-quality telecommunication services to passengers in Grand Paris Express stations and inside trains. To that purpose, Société du Grand Paris is interested in developing new technologies such as mobile relays. A testbed using real radio transmissions has already proved that a mobile relay architecture can be easily implemented with standard Evolved Packet Core (EPC) and with full-compatibility with 3GPP recommendations [3].

In railway cellular networks, base stations are regularly placed along railway sections. With LTE, cooperation between base stations is made possible. In this paper, we consider mobile railway communications assuming that each carriage is equipped with one mobile eNodeB station. The mobile eNodeB onboard serves as relay of passengers' communications towards the cellular base stations along the section. We also impose that it communicates with both base stations

This work has been financially supported by Société du Grand Paris. 978-1-7281-4490-0/20/\$31.00 @ 2020 IEEE at both ends of the section and that these two base stations cooperate. More precisely, we study the uplink with receive macrodiversity [4]. We assume that the carriage is equipped with two distinct multiple antenna arrays, part of two remote radio heads (RRH) connected to the same onboard baseband unit (BBU). Our purpose is the design of distributed coding schemes between back and front antenna arrays that take advantage of macrodiversity to maintain the quality of service as the carriage moves along the section.

Our contributions are two-fold. First, we show how much macrodiversity through cooperation of neighbouring base stations along the railway section is efficient to ensure transmission robustness, provided the eNodeB onboard communicates with both of them. Second is the definition of two MIMO coding schemes involving both carriage antenna arrays. One, based on Alamouti coding [5] principle, maximizes the diversity gain at the expense of the throughput and data traffic on CPRI [6] [7] links. The other, inspired by the Golden code [8], maximizes the trade-off between throughput and error rate.

The paper is organized as follows. Section II describes the system model and the reference scheme based on spatial multiplexing and transmit antenna selection. Section III introduces first proposed scheme based on Alamouti coding principle and derives analytical expressions of throughput and error rate. Section IV defines the second proposed scheme inspired by the Golden code, with analytical study of its performance. Section $\mathrm{V}$ is dedicated to simulations and supports the theoretical analysis. Section VI concludes the paper.

\section{A. Notations}

$x$ in bold font and $x$ in normal font stand for a vector and a scalar respectively. Given an $N \times M$ complex-valued matrix $\boldsymbol{A}$, we denote by $\boldsymbol{A}^{T}, \boldsymbol{A}^{H}, \boldsymbol{A}^{*}$ its transpose, its conjugate transpose and its conjugate respectively. $\boldsymbol{A}_{i}$ is its $i$-th column. The norm $\|\boldsymbol{A}\|$ is defined as the Frobenius norm by $\sqrt{\operatorname{tr}\left(\boldsymbol{A} \boldsymbol{A}^{H}\right)}$ with $\operatorname{tr}($.$) the trace operator. \mathbf{0}_{N}$ denotes the length- $N$ all-zero column vector.

The complementary error function is defined by $\operatorname{erfc}(x)=$ $\frac{2}{\sqrt{\pi}} \int_{x}^{+\infty} \exp \left(-t^{2}\right) d t$. 


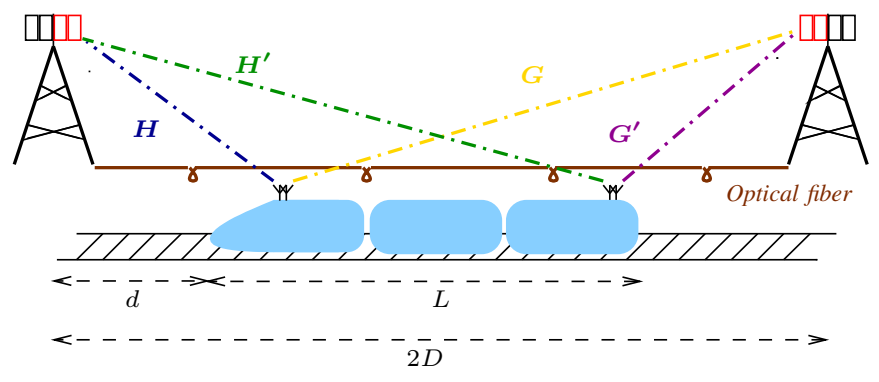

Fig. 1: System model and notations

\section{SYSTEM MODEL, ASSUMPTIONS AND PURE MACRODIVERSITY SCHEME}

We consider the uplink of a railway communication system between a carriage moving on a railway section and two base stations located at each end of the section. We assume that the downlink does not interfere with the uplink (frequency or time division duplexing). Furthermore, orthogonal frequency division multiple access (OFDMA) is applied for resource allocation in such a manner that inter-cell and intra-cell interference can be neglected. Without loss of generality, we assume that the carriage moves from left to right.

Each base station (BTS) is equipped with two sector antenna arrays (respective coverage area on either side of the BTS). BTSs are assumed to be either interconnected or connected to a remote BTS controller thanks to error-free optical fiber links. Receive cooperation is thus possible. We denote by $N$ each BTS antenna array element number.

The carriage is equipped with two omnidirectional antenna arrays (one on the front, one on the back), part of a remote radio head (RRH). Both RRH are connected through a common public radio interface (CPRI) [6], [7] to the same baseband unit (BBU), which orchestrates transmissions from the carriage. We denote by $M$ the antenna number on each side of the carriage.

\section{A. System model}

We consider a subcarrier index $p$. Let $\boldsymbol{H}$ and $\boldsymbol{G}$ stand for the subchannel matrix between the front of the carriage and the BTS on the left and on the right, respectively. Let $\boldsymbol{H}^{\prime}$ and $G^{\prime}$ stand for the equivalent notations for the back of the carriage (cf. Fig. 1). The BTS on the left and on the right are referred by the index "L" and "R", respectively. We assume that the channel variation between two adjacent subcarriers is negligible.

The transmitted vectors from the front and the back of the carriage are denoted by $\boldsymbol{x}(p)$ and $\boldsymbol{x}^{\prime}(p)$, respectively. Then the received vectors at the left and at the right BTS are denoted by $\boldsymbol{y}_{\boldsymbol{L}}(p)$ and $\boldsymbol{y}_{\boldsymbol{R}}(p)$, respectively.

The transmission can be modeled by

$$
\begin{aligned}
\boldsymbol{y}_{\boldsymbol{L}}(p) & =\boldsymbol{H} \boldsymbol{x}(p)+\boldsymbol{H}^{\prime} \boldsymbol{x}^{\prime}(p)+\boldsymbol{n}_{\boldsymbol{L}}(p), \\
\boldsymbol{y}_{\boldsymbol{R}}(p) & =\boldsymbol{G} \boldsymbol{x}(p)+\boldsymbol{G}^{\prime} \boldsymbol{x}^{\prime}(p)+\boldsymbol{n}_{\boldsymbol{R}}(p),
\end{aligned}
$$

where $\boldsymbol{n}_{\boldsymbol{L}}(p)$ and $\boldsymbol{n}_{\boldsymbol{R}}(p)$ are noise vectors with independent identically distributed (i.i.d.) components. We consider complex zero-mean circularly symmetric Gaussian distribution with variance $\sigma_{n}^{2}$.

First macrodiversity level comes from the joint use of both $\boldsymbol{y}_{\boldsymbol{L}}(p)$ and $\boldsymbol{y}_{\boldsymbol{R}}(p)$ to detect $\boldsymbol{x}(p)$ and $\boldsymbol{x}^{\prime}(p)$. The key principle is the following one. As the carriage moves along the section from left to right, the average signal to noise ratio on $\boldsymbol{y}_{\boldsymbol{L}}(p)$ will almost surely decrease while the average signal to noise ratio on $\boldsymbol{y}_{\boldsymbol{R}}(p)$ will almost surely increase. This phenomenon is expected to avoid high variations of the transmission quality and data rate during the communication.

\section{B. Design constraints}

Our purpose is to design a macrodiversity transmission scheme which benefits from additional existing diversity provided by the cooperation between the two carriage antenna arrays.

Indeed, due to the shadowing resulting from the interference from another carriage coming the other way or simply from the antenna array on the other side, transmission from one antenna array of the carriage may experience more severe propagation conditions than the other one. Joint coding between both carriage antenna arrays is a way to further enhance the robustness of the transmission as the carriage moves along the section.

Taking into account the train speed and the double railway channel selectivity, open-loop schemes with limited required channel state information knowledge may be better suited.

To reduce the data traffic on CPRI links from BBU to RRH, we apply antenna selection [9] at both sides of the carriage. Another advantage of antenna selection is its robustness towards correlation between antenna array elements.

In the following, without loss of generality we denote by $\boldsymbol{h}, \boldsymbol{h}^{\prime}, \boldsymbol{g}$ and $\boldsymbol{g}^{\prime}$ the resulting equivalent subchannel vectors. For example, $\boldsymbol{h}$ is defined by

$$
\boldsymbol{h}=\arg \max _{\substack{\boldsymbol{H}_{i} \\ 1 \leq i \leq M}} \boldsymbol{H}_{i}^{H} \boldsymbol{H}_{i}
$$

The equivalent transmission model is thus given by

$$
\begin{aligned}
& \boldsymbol{y}_{\boldsymbol{L}}(p)=\boldsymbol{h} x(p)+\boldsymbol{h}^{\prime} x^{\prime}(p)+\boldsymbol{n}_{\boldsymbol{L}}(p), \\
& \boldsymbol{y}_{\boldsymbol{R}}(p)=\boldsymbol{g} x(p)+\boldsymbol{g}^{\prime} x^{\prime}(p)+\boldsymbol{n}_{\boldsymbol{R}}(p),
\end{aligned}
$$

where $x(p)$ and $x^{\prime}(p)$ are now scalars.

\section{Pure macrodiversity scheme}

Pure macrodiversity scheme does not apply joint coding between carriage antenna arrays. It consists in transmitting two independent streams from the front and the back of the carriage (spatial multiplexing). It also maximizes the data throughput. But the main drawback is the limited diversity gain.

The schemes proposed in this paper are based on the use of two adjacent subcarriers. To set a generic formalisation, we will describe the pure macrodiversity scheme (referred to as "M") from two adjacent subcarriers. The pure macrodiversity scheme is defined by

$$
\boldsymbol{X}_{M}(p)=\left(\begin{array}{cc}
x(p) & x(p+1) \\
x^{\prime}(p) & x^{\prime}(p+1)
\end{array}\right)=\left(\begin{array}{cc}
s_{4 p} & s_{4 p+2} \\
s_{4 p+1} & s_{4 p+3}
\end{array}\right) .
$$


The receiver uses the observations collected by both BTS antenna arrays and corresponding to the pair of adjacent subcarriers $(p, p+1)$.

Let $\boldsymbol{y}_{. .}=\left(\boldsymbol{y}_{\text {.. }}(p) \quad \boldsymbol{y}_{\text {.. }}(p+1)\right)^{T}$ with ".." standing for " $L "$ or " $R$ ", and $\boldsymbol{y}=\left(\begin{array}{ll}\boldsymbol{y}_{\boldsymbol{L}}^{T} & \boldsymbol{y}_{\boldsymbol{R}}^{T}\end{array}\right)$. We define $\boldsymbol{n}_{\boldsymbol{L}}, \boldsymbol{n}_{\boldsymbol{R}}$ and $\boldsymbol{n}$ in the same way. Then an equivalent transmission model reads

$$
\boldsymbol{y}=\boldsymbol{F} \boldsymbol{s}+\boldsymbol{n},
$$

where $\boldsymbol{F}$ is given by

$$
\boldsymbol{F}=\left(\begin{array}{cccc}
\boldsymbol{h} & \boldsymbol{h}^{\prime} & \mathbf{0}_{N} & \mathbf{0}_{N} \\
\mathbf{0}_{N} & \mathbf{0}_{N} & \boldsymbol{h} & \boldsymbol{h}^{\prime} \\
\boldsymbol{g} & \boldsymbol{g}^{\prime} & \mathbf{0}_{N} & \mathbf{0}_{N} \\
\mathbf{0}_{N} & \mathbf{0}_{N} & \boldsymbol{g} & \boldsymbol{g}^{\prime}
\end{array}\right) .
$$

and $\boldsymbol{s}=\left(\begin{array}{llll}s_{4 p} & s_{4 p+1} & s_{4 p+2} & s_{4 p+3}\end{array}\right)^{T}$ is the information vector.

Maximum-likelihood (ML) detection is achieved by minimization of $\|\boldsymbol{y}-\boldsymbol{F} \boldsymbol{s}\|$ over all realizations of $\boldsymbol{s}$.

According to (7) and, assuming that $\mathrm{E}\left[\boldsymbol{s s ^ { H }}\right]=\sigma_{s}^{2} \boldsymbol{I}_{4}$, the maximum achievable throughput per subcarrier is given by

$$
I_{M}=\frac{1}{2} \log _{2} \operatorname{det}\left(\mathrm{I}_{4}+\frac{\sigma_{s}^{2}}{\sigma_{n}^{2}} \boldsymbol{F}^{H} \boldsymbol{F}\right) .
$$

Let us define $\mu=\boldsymbol{h}^{H} \boldsymbol{h}+\boldsymbol{g}^{H} \boldsymbol{g}, \mu^{\prime}=\boldsymbol{h}^{{ }^{H}} \boldsymbol{h}^{\prime}+\boldsymbol{g}^{\prime H} \boldsymbol{g}^{\prime}, \gamma=$ $\mu+\mu^{\prime}$ and $\theta=\boldsymbol{h}^{H} \boldsymbol{h}^{\prime}+\boldsymbol{g}^{H} \boldsymbol{g}^{\prime}$. Then immediate computations yield

$$
I_{M}=\log _{2}\left(1+\frac{\sigma_{s}^{2}}{\sigma_{n}^{2}} \gamma+\frac{\sigma_{s}^{4}}{\sigma_{n}^{4}} \mu \mu^{\prime}-|\theta|^{2}\right) .
$$

Let us mention that without macrodiversity, assuming for instance the communication with only the BTS on the left, we would have $\gamma=\mu, \mu^{\prime}=0$ and $\theta=0$, yielding to a degraded throughput, particularly when the carriage moves away.

The average maximum achievable throughput $\bar{I}_{M}$ is obtained by averaging $I_{M}$ over the realizations of the channel.

\section{First Distributed SCHEME BASED ON AlAmouti CODE}

Alamouti code [5] is used to define open-loop transmission diversity schemes in most communication standards due to its maximum diversity gain and optimal low-complexity linear detection. We refer to the proposed scheme as scheme A.

\section{A. Proposed scheme A}

Scheme A enables to only transmit two different modulation symbols per subcarrier couple.

Denoting by $s=\left(\begin{array}{ll}s_{2 p} & s_{2 p+1}\end{array}\right)^{T}$ the information data vector, proposed scheme $\mathrm{A}$ is defined by

$$
\boldsymbol{X}_{A}(p)=\left(\begin{array}{cc}
s_{2 p} & s_{2 p+1}^{*} \\
s_{2 p+1} & -s_{2 p}^{*}
\end{array}\right) .
$$

$$
\text { Let } \tilde{\boldsymbol{H}}=\left(\begin{array}{cc}
\boldsymbol{h}^{H} & -\boldsymbol{h}^{\prime T} \\
\boldsymbol{h}^{\prime H} & \boldsymbol{h}^{T}
\end{array}\right), \tilde{\boldsymbol{G}}=\left(\begin{array}{cc}
\boldsymbol{g}^{H} & -\boldsymbol{g}^{T} \\
\boldsymbol{g}^{\prime H} & \boldsymbol{g}^{T}
\end{array}\right) \text {. }
$$

BTS cooperation is done through joint processing of all observations collected by their antenna array elements.

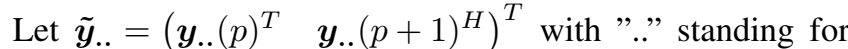
" $L "$ or " $R$ ". We define $\tilde{\boldsymbol{n}}_{\boldsymbol{L}}$ and $\tilde{\boldsymbol{n}}_{\boldsymbol{R}}$ in the same way.

The optimum ML-detection output is thus obtained by

$$
\tilde{\boldsymbol{s}}=\left(\begin{array}{c}
\tilde{s}_{2 p} \\
\tilde{s}_{2 p+1}
\end{array}\right)=\tilde{\boldsymbol{H}} \tilde{\boldsymbol{y}}_{\boldsymbol{L}}+\tilde{\boldsymbol{G}} \tilde{\boldsymbol{y}}_{\boldsymbol{R}}
$$

\section{B. Performance analysis}

Immediate computations yield an equivalent writing of $\tilde{s}$, which is

$$
\tilde{\boldsymbol{s}}=\gamma \boldsymbol{s}+\tilde{\boldsymbol{n}},
$$

where $\tilde{\boldsymbol{n}}=\tilde{\boldsymbol{H}} \tilde{\boldsymbol{n}}_{\boldsymbol{L}}+\tilde{\boldsymbol{G}} \tilde{\boldsymbol{n}}_{\boldsymbol{R}}$. Components of $\tilde{\boldsymbol{n}}$ are i.i.d., complex circularly symmetric Gaussian with zero mean and variance equal to $\gamma \sigma_{n}^{2}$.

The output detection SNR equals $S N R_{A}=\gamma \frac{\sigma_{s}^{2}}{\sigma^{2}}$, which proves that this scheme maximizes the diversity gain.

Given the channel state, the maximum achievable throughput per subcarrier is given by

$$
I_{A}=\log _{2}\left(1+\gamma \frac{\sigma_{s}^{2}}{\sigma_{n}^{2}}\right) .
$$

For a QPSK modulation with Gray mapping, given the channel state, the binary error probability equals

$$
P_{e, A}=\frac{1}{2} \operatorname{erfc}\left(\sqrt{\frac{\gamma}{2} \frac{\sigma_{s}^{2}}{\sigma_{n}^{2}}}\right)
$$

The average maximum achievable throughput $\bar{I}_{A}$ and the average binary error probability $\bar{P}_{e, A}$ are obtained by averaging $I_{A}$ and $P_{e, A}$ over the realizations of the channel.

\section{SECOND DISTRIBUTED CODING SCHEME BASED ON GOLDEN CODE}

To reduce the data traffic on CPRI links within the carriage, we propose to design a second scheme based on the Golden code [8]. The Golden code achieves the maximum diversitymultiplexing trade-off. It maximises the diversity gain as the Alamouti code and the data rate as spatial multiplexing [8]. To the best of our knowledge, its main drawback is the sensitivity of its performance towards quantization [10]. In the remaining of the paper, we refer to the proposed scheme based on Golden code as scheme $\mathrm{G}$.

\section{A. Proposed scheme $G$}

Let us introduce the defining parameters as $g=\frac{-1+\sqrt{5}}{2}$, $a=\frac{1}{\sqrt{1+g^{2}}}$ and $b=\frac{g}{\sqrt{1+g^{2}}}$. The power normalization is ensured by $a^{2}+b^{2}=1$.

Denoting by $s=\left(\begin{array}{llll}s_{4 p} & s_{4 p+1} & s_{4 p+2} & s_{4 p+3}\end{array}\right)^{T}$ the information data vector, we define the second macrodiversity scheme by

$$
\boldsymbol{X}_{G}(p)=\left(\begin{array}{cc}
a s_{4 p}+i b s_{4 p+1} & a s_{4 p+2}-b s_{4 p+3} \\
b s_{4 p+2}+a s_{4 p+3} & i b s_{4 p}+a s_{4 p+1}
\end{array}\right) .
$$

Compared to scheme A, scheme $\mathrm{G}$ (likewise scheme $\mathrm{M}$ ) enables to transmit twice more symbols per channel use, which will enable to divide by two the traffic on the CPRI links, while enhancing the throughput. 


\section{B. Performance analysis}

Using the same notations as in Section II-C, we derive an equivalent transmission model, which reads

$$
\boldsymbol{y}=\boldsymbol{F Q} \boldsymbol{s}+\boldsymbol{n},
$$

where $Q$ is given by

$$
\boldsymbol{Q}=\left(\begin{array}{cccc}
a & i b & 0 & 0 \\
0 & 0 & b & a \\
0 & 0 & a & -b \\
i b & a & 0 & 0
\end{array}\right) .
$$

As $Q$ is unitary, the maximum achievable throughput per subcarrier is the same as for scheme $\mathrm{M}$, namely

$$
I_{G}=\log _{2}\left(1+\frac{\sigma_{s}^{2}}{\sigma_{n}^{2}} \gamma+\frac{\sigma_{s}^{4}}{\sigma_{n}^{4}} \mu \mu^{\prime}-|\theta|^{2}\right) .
$$

\section{Simulations}

\section{A. Channel model}

The section length is equal to $2 D$ with $D=400$ meters, while the carriage length $L$ is fixed to 100 meters. $d$ is defined as the distance between left BTS and the front of carriage.

We consider $\boldsymbol{H}=\beta \boldsymbol{H}_{N}, \boldsymbol{H}^{\prime}=\lambda \boldsymbol{H}_{N}^{\prime}, \boldsymbol{G}=\lambda^{\prime} \boldsymbol{G}_{N}$ and $\boldsymbol{G}^{\prime}=\beta^{\prime} \boldsymbol{G}_{N}^{\prime}$ where $\boldsymbol{H}_{N}, \boldsymbol{H}_{N}^{\prime}, \boldsymbol{G}_{N}$ and $\boldsymbol{G}_{N}^{\prime}$ are i.i.d. such that their components are i.i.d. complex symmetric Gaussian with zero mean and unitary variance (non-correlated flat Rayleigh fading channel). $\beta, \lambda, \beta^{\prime}$ et $\lambda^{\prime}$ are fixed thanks to the Friis formula in free-space with shadowing parameter $\ell$ such that:

$$
\begin{aligned}
\beta & =\frac{2 D-L}{2 d}, \\
\beta^{\prime} & =\frac{2 D-L}{4 D-2 d-2 L}, \\
\lambda & =\sqrt{\ell} \frac{2 D-L}{2 d+2 L}, \\
\lambda^{\prime} & =\sqrt{\ell} \frac{2 D-L}{4 D-2 d} .
\end{aligned}
$$

The shadowing parameter $\ell$ corresponds to the interference due to another carriage coming the other way or due to the other antenna array. When the carriage moves from right to left, $\beta$ and $\gamma$ increase while $\beta^{\prime}$ and $\gamma^{\prime}$ decrease.

The performance will be studied according to a target signal to noise ratio, corresponding to a full shadowing and the carriage located in the middle of the section (worst case). We define the relative position of the carriage center by $\Delta=\frac{2 d+L}{4 D} . \Delta=0.5$ means that it is located in the middle.

\section{B. Macrodiversity impact}

Figures 2 and 3 give the performance of scheme A with and without macrodiversity in terms of binary error rate (BER) and maximum achievable throughput per subcarrier for $3 \mathrm{~dB}$ of shadowing and different relative positions. With macrodiversity, $M=2,4$ and $N=2$. Without macrodiversity, $M=2$ and $N=2,4$. We observe that macrodiversity enables to maintain both metrics within a given range as the carriage moves away. Increasing the transmit antenna number further improves performance, without much additional complexity, as antenna selection is applied. In the absence of macrodiversity, performance significantly degrades as $\Delta$ gets higher, and the loss can be reduced by increasing the receive antenna number.

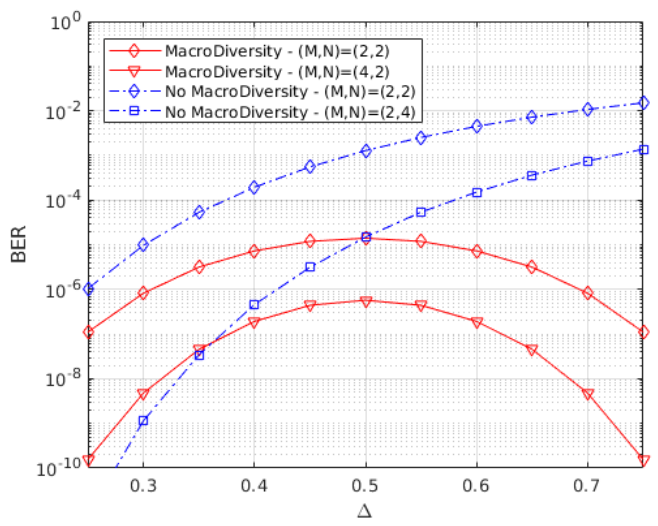

Fig. 2: Impact of macrodiversity on the error rate as a function of $\Delta$. Scheme A, QPSK, shadowing of $3 \mathrm{~dB}$

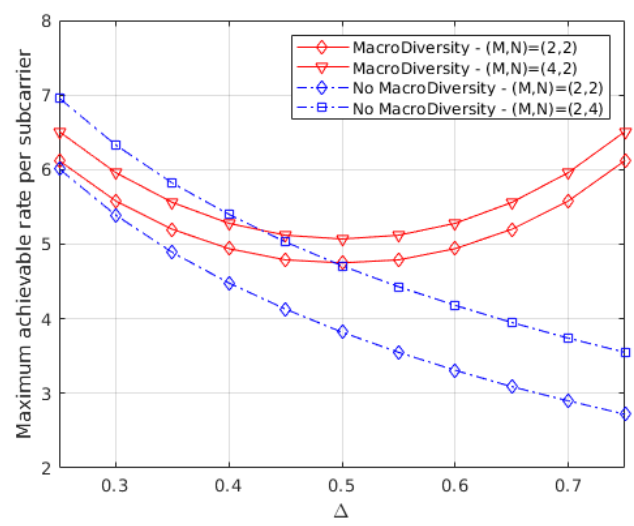

Fig. 3: Impact of macrodiversity on the maximum achievable rate per subcarrier as a function of $\Delta$. Scheme A, shadowing of $3 \mathrm{~dB}$

C. Comparison of the proposed distributed coding schemes with receive macrodiversity

Performance in terms of BER and maximum achievable throughput per subcarrier for $3 \mathrm{~dB}$ of shadowing, for schemes $\mathrm{A}, \mathrm{G}$ and $\mathrm{M}$ are plotted in Figures 4, 5, 6 and 7. We remind that scheme A transmits half the number of symbols compared to schemes $\mathrm{G}$ and $\mathrm{M}$, which accounts for its superiority (resp. inferiority) in terms of BER (resp. maximum throughput). As expected, scheme $G$ performs the same as scheme $M$ in terms of throughput and much better in terms of BER. The slopes of BER curves are the same for $\mathrm{A}$ and $\mathrm{G}$ and worse for $\mathrm{M}$. This is in agreement with the theory: scheme $G$ achieves the same diversity as scheme A. Given a BER value, the differences between the schemes decrease as the carriage moves to the 
middle of the section (minimum value). As for the throughput, the gain of $\mathrm{G}$ and $\mathrm{M}$ over $\mathrm{A}$ is significant: around 5 and 4 additional bits per subcarrier for a target SNR of $12 \mathrm{~dB}$ with $\Delta=0.25$ and $\Delta=0.5$, respectively.

\section{CONCLUSION}

In this paper, we have considered future mobile railway communication systems with onboard eNodeB serving as relays of passengers' communications and connected with both cooperating base stations at the ends of the railway section. We have proposed distributed coding schemes with receive macrodiversity. Simulations have shown the efficiency of macrodiversity on one hand, and of the distributed coding scheme based on the Golden code on the other hand, to maintain high quality and high thoughput with reduced data traffic on CPRI links as the carriage moves along the section. Future work will deal with tests in real environment.

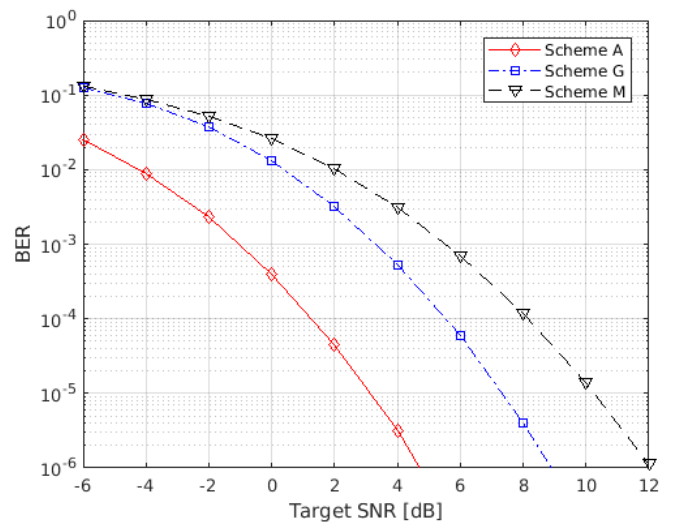

Fig. 4: Comparison of proposed distributed coding schemes with receive macrodiversity. $(M, N)=(2,2)$, QPSK, $\Delta=$ 0.25

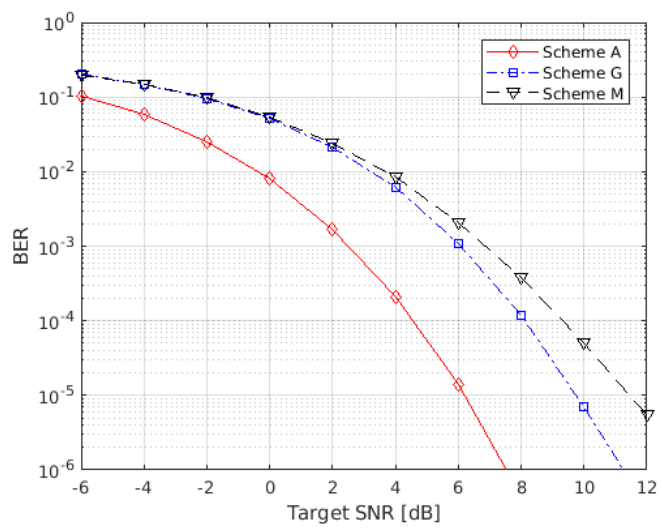

Fig. 5: Comparison of proposed distributed coding schemes with receive macrodiversity. $(M, N)=(2,2)$, QPSK, $\Delta=0.5$

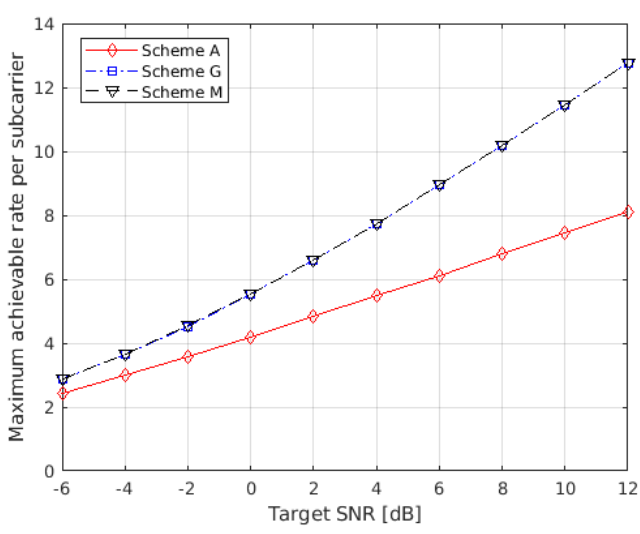

Fig. 6: Comparison of proposed distributed coding schemes with receive macrodiversity. $(M, N)=(2,2), \Delta=0.25$

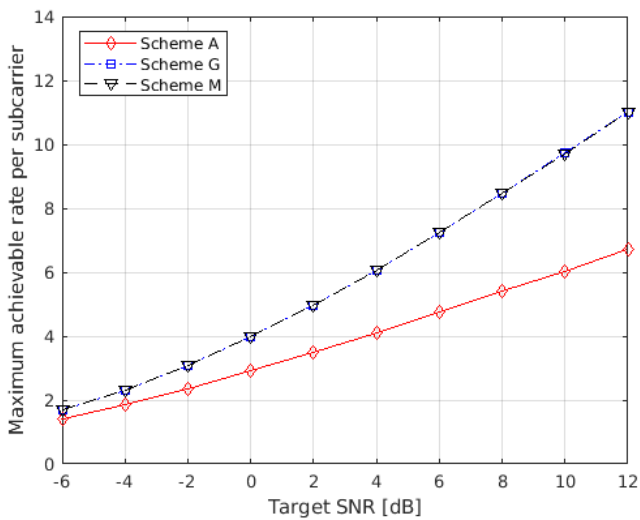

Fig. 7: Comparison of proposed distributed coding schemes with receive macrodiversity. $(M, N)=(2,2), \Delta=0.5$

\section{REFERENCES}

[1] R. Chen, W-X. Long, G. Mao and C. Li, "Development Trends of Mobile Communication Systems for railways", IEEE Communications Surveys and Tutorials, Fourth Quarter 2018

[2] Société du Grand Paris, île de France mobilités and Grand Paris express, "Matériels roulants du Grand Paris Express : Le marché entre dans son ultime phase", May2018, Press release

[3] T. Kerdoncuff, T. Galezowski and X. Lagrange, "Mobile relay for LTE: proof of concept and performance measurements", IEEE 87th Vehicular Technology Conference, July 2018

[4] I. Rivas, L.J. Ibbetson and L.B. Lopes, Macrodiversity reception performance investigation in microcellular networks, IEEE 47th Vehicular Technology Conference. Technology in Motion, May 1997

[5] S.M. Alamouti, "A simple transmit diversity technique for wireless communications", IEEE Journal on Selected Areas in Communications, October, 1998, vol. 16, no. 8, pp. 1451-1458

[6] Ericsson AB, Huawei Technologies Co. Ltd and NEC Corporation and Alcatel Lucent and Nokia Networks,'Common Public Radio Interface (CbPRI); Interface SpecificationV7.0, October,2015

[7] A. de la Oliva, J.A. Hernandez, D. Larrabeiti and A. Azcorra, "An Overview of the CPRI Specification and its Application to C-RANBased LTE Scenarios", IEEE Communications Magazine, February 2016, vol. 54, no. 5, pp.152-159

[8] J-C. Belfiore, G. Rekaya and E. Viterbo, "The Golden Code: A 22 Full-Rate Space-Time Code with Non-Vanishing Determinants", IEEE Transactions on Information Theory, April 2005, vol. 51, no. 4, pp. 1432-1436 
[9] R.S. Blum and J.H. Winters, "On Optimum MIMO With Antenna Selection", IEEE Communication Letters, August 2002, vol. 6, no. 8, pp. 322-324

[10] J. Harshan and E. Viterbo, "On the Robustness of Algebraic STBCs to Coefficient Quantization", IEEE Australian Communications Theory Workshop, February 2012, pp.55-60 